\title{
Exotic Order in Simple Models of Bosonic Systems
}

\author{
O. I. Motrunich and T. Senthil \\ Massachusetts Institute of Technology, 77 Massachusetts Avenue, Cambridge, Massachusetts 02139
}

(Received 15 May 2002; published 20 December 2002)

\begin{abstract}
We show that simple Bose Hubbard models with unfrustrated hopping and short range two-body repulsive interactions can support stable fractionalized phases in two and higher dimensions, and in zero magnetic field. The simplicity of the constructed models advances the possibility of a controlled experimental realization and novel applications of such unconventional states.
\end{abstract}

DOI: $10.1103 /$ PhysRevLett.89.277004

PACS numbers: 74.20.Mn, 71.10.Fd, 71.10.Hf

Recent theoretical developments [1-4] have shown that two- or three-dimensional strongly correlated systems in zero magnetic field could display quantum phases with fractional quantum numbers. This theoretical progress, inspired mostly by the search for a theory of the hightemperature superconductors [5], is likely to play an important role in our eventual understanding of the mysterious properties of several strongly interacting electronic systems. However, until now, no such experimental system has been unambiguously shown to display fractional quantum numbers. Further impetus for the search for experimental realizations of fractionalization comes from the possibility of using such states to construct qubits [6,7]. The topological structure inherent in these states naturally protects the system from decoherence.

The primary goal of this paper is the identification and possible design of specific condensed matter systems which display the phenomenon of fractional quantum numbers. To that end, we study particularly simple models of bosons with unfrustrated hopping and short ranged two-body repulsive interactions on a two-dimensional (2D) square lattice. We show that, in particular parameter ranges, a fractionalized insulating phase exists where there are excitations whose charge is one-half that of the underlying bosons. Superfluid or more conventional insulating phases result in other parameter ranges. The simplicity of our models opens up the possibility that they can be realized in arrays of quantum Josephson junctions, or possibly in ultracold atomic gases. This would provide a definite experimental realization of a fractionalized phase which could then possibly be exploited to construct topologically protected qubits.

The fractionalized phase appears in a region of intermediate correlations where neither the boson kinetic energy nor repulsive potential energy completely dominates over the other. This lends support to the general notion that fractionalization is to be looked for in a many-body system at intermediate correlations. For example, in the interacting electron system, fractionalization possibly occurs at intermediate values of density somewhere between the extreme low density Wigner crystal and the high density Fermi liquid regimes. Similarly, electronic
Mott insulators that are close to the metal-insulator transition may be good candidates for fractionalization.

The generalization of our models to three dimensions (3D) is of some interest. The $3 \mathrm{D}$ version of our boson Hubbard model has in fact two distinct fractionalized insulating phases: First, there is a fractionalized phase similar to the one in $2 \mathrm{D}$, with the distinct excitations being a charge- $1 / 2$ chargon and a $Z_{2}$ vortex (vison) line. The topological order in this phase is stable up to a finite nonzero temperature. Experimental realization of this phase may therefore be of interest for the quantum computing application as a way of controlling errors due to nonzero temperature. Another distinct fractionalized insulator also appears in 3D. In this phase, the excitations are a gapped charge- $1 / 2$ chargon, a gapless linear dispersing "photon," and a gapped topological point defect (the "monopole"). Wen [8] has recently pointed out that stable mean field theories may be constructed for quantum phases where a massless $U(1)$ gauge boson (a photon) emerges in the low-energy description. Our results provide an explicit and concrete model for such a phase.

Fractionalization of bosons in two dimensions.-Consider bosons moving on the lattice shown in Fig. 1. A physical realization may be a Josephson junction array

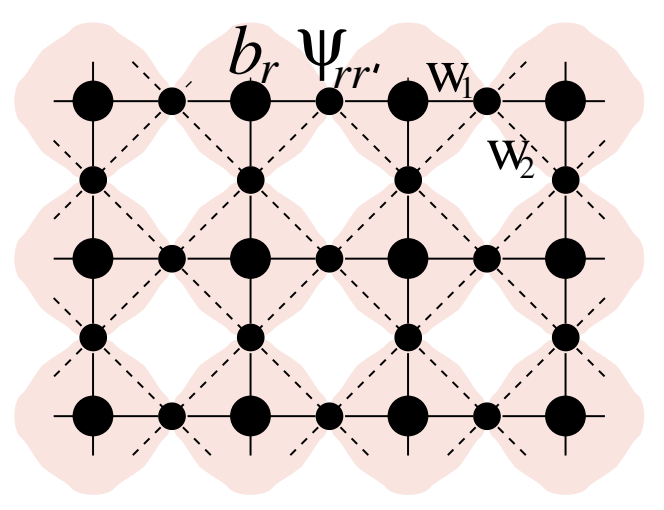

FIG. 1 (color online). Josephson junction array on a 2D bondcentered square lattice modeled by the Hamiltonian Eq. (1). Each shaded area indicates schematically cluster charging energy $U N_{r}^{2}$. 
with superconducting islands arranged on the sites of the "bond-centered" square lattice and Josephson coupled with each other as indicated by the links. We also stipulate repulsive interactions between the bosons ("charging energy") that favors charge neutrality not only on individual islands but also on the shaded clusters (note that neighboring clusters share one site) [9]. The corresponding Hamiltonian is

$$
\begin{aligned}
H= & -w_{1} \sum_{r, r^{\prime} \in r}\left(b_{r}^{\dagger} \psi_{r r^{\prime}}+\text { H.c. }\right) \\
& -w_{2} \sum_{\left[r r^{\prime} r^{\prime \prime}\right]}\left(\psi_{r r^{\prime}}^{\dagger} \psi_{r^{\prime} r^{\prime \prime}}+\text { H.c. }\right)+u_{b} \sum_{r}\left(n_{r}^{b}\right)^{2} \\
& +u_{\psi} \sum_{\left\langle r r^{\prime}\right\rangle}^{\psi}\left(n_{r r^{\prime}}^{\psi}\right)^{2}+U \sum_{r} N_{r}^{2} .
\end{aligned}
$$

Here, $b_{r}^{\dagger}=e^{i \theta_{r}}$ represent bosons (Cooper pairs) residing on the corner sites of the lattice, and $\psi_{r r^{\prime}}^{\dagger}=e^{i \phi_{r r^{\prime}}}$ represent bosons on the bond-centered sites (identified by the bond end points); $n_{r}^{b}, n_{r r^{\prime}}^{\psi}$ are the corresponding boson numbers, $\left[\theta_{r}, n_{r}^{b}\right]=i$, and similarly for the $\psi$ bosons. Throughout, we work with a number-phase (quantum rotor) representation of the bosons, as is particularly appropriate in the Josephson junction array realization [10].

The $w_{1}$ term is a boson hopping (Josephson coupling) between the corner and the bond-centered sites, and $r^{\prime} \in r$ sums over all such bonds emanating from $r$. The $w_{2}$ term is a boson hopping between the neighboring bond-centered sites as indicated with dashed lines in Fig. 1. The $u_{b}$ and $u_{\psi}$ terms represent on-site boson repulsion, while the $U$ term is the cluster charging energy that favors charge neutrality in each cluster. The operator $N_{r}$ associated with each cluster is defined through

$$
N_{r}=2 n_{r}^{b}+\sum_{r^{\prime} \in r} n_{r r^{\prime}}^{\psi}
$$

The total boson number of the system is $N_{\text {tot }}=\frac{1}{2} \sum_{r} N_{r}$. Both the $b$ bosons and the $\psi$ bosons are assigned charge $q_{b}$. The model has only a global $U(1)$ charge conservation symmetry.

For large $w_{1}, w_{2} \gg u_{b}, u_{\psi}, U$ the system is a superfluid. In the opposite limit, $u_{b}, u_{\psi}, U \gg w_{1}, w_{2}$, the system is a conventional Mott insulator with charge quantized in units of $q_{b}$. We argue below that when the charging energies $U$ and $u_{b}, u_{\psi}$ are varied separately, there is an intermediate regime $U \gg w_{1}, w_{2} \gg \sqrt{u_{b} \bar{U}}, \sqrt{u_{\psi} \bar{U}}$, in which the system is a stable fractionalized insulator with charge $q_{b} / 2$ excitations and charge 0 visons above a ground state with no conventional broken symmetries. A schematic phase diagram of our model is shown in Fig. 2.

The analysis in the limit of large cluster interaction $U \gg w_{1}, w_{2}, u_{b}, u_{\psi}$ is similar to that in the large $U$ limit of the electronic Hubbard model at half filling. If the other terms are all zero, there is a degenerate manifold of ground states specified by the requirement $N_{r}=0$ for each $r$ (recall that the operators $n_{r}^{b}$ and $n_{r r^{\prime}}^{\psi}$ are defined as conjugates of the corresponding phase variables and

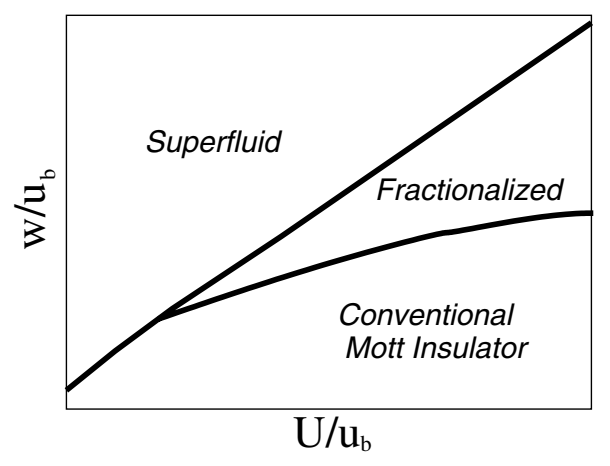

FIG. 2. Schematic phase diagram of the boson Hubbard model Eq. (1) for a particular cut $w \equiv w_{1} \simeq w_{2}$ and $u_{b} \simeq u_{\psi}$ through the parameter space.

have eigenvalues that can take all integer values including negative ones; thus, the constrained Hilbert space $N_{r}=0$ is indeed nontrivial). This ground state sector is separated by a large charge gap $U$ from the nearest sectors. Including the $w_{1}, w_{2}, u_{b}, u_{\psi}$ terms lifts the degeneracy in each such zeroth-order sector, and this is best described by deriving the corresponding effective Hamiltonians for small perturbing couplings.

Consider the ground state sector $N_{r}=0$ for all $r$. An elementary calculation gives

$$
\begin{aligned}
H_{\mathrm{eff}}^{(0)}= & H_{u_{b}, u_{\psi}}-J_{\text {bond }} \sum_{\left\langle r r^{\prime}\right\rangle}\left[\left(\psi_{r r^{\prime}}^{\dagger}\right)^{2} b_{r} b_{r^{\prime}}+\text { H.c. }\right] \\
& -K_{\text {ring }} \sum_{\square}\left(\psi_{12}^{\dagger} \psi_{23} \psi_{34}^{\dagger} \psi_{41}+\text { H.c. }\right),
\end{aligned}
$$

where $H_{u_{b}, u_{\psi}}$ stands for the on-site repulsion terms as in Eq. (1), $J_{\text {bond }}=w_{1}^{2} / U$, and $K_{\text {ring }}=2 w_{2}^{2} / U$.

A simple change of variables shows [4] that $H_{\mathrm{eff}}^{(0)}$ together with the constraint $N_{r}=0$ can be regarded as the well-studied [11] $(2+1) \mathrm{D}$ compact $U(1)$ gauge theory coupled to a charge 2 scalar field. In $(2+1) \mathrm{D}$, there are two distinct phases shown in Fig. 3 . For $J_{\text {bond }}, K_{\text {ring }} \lesssim u_{b}$, $u_{\psi}$, the gauge theory is "confined," and all excitations carrying nonzero "gauge charge" are confined. Zero gauge charge excitations carrying physical charge quantized in units of $q_{b}$ of course exist with a gap of order $2 U$. This is the conventional Mott insulator of our boson model.

In the opposite regime, $J_{\text {bond }}, K_{\text {ring }} \gtrsim u_{b}$, $u_{\psi}$, the gauge theory is in the "deconfined Higgs" phase. Objects with $N_{r}=1$ at some site, i.e., physical charge $q_{b} / 2$ (chargon), have gauge charge 1 , are not confined, and can propagate above a finite gap of order $U$. There is also a stable gapped $Z_{2}$ vortex excitation (vison). The deconfined phase has a topological order $[12,13]$ : e.g., the ground state is twofold degenerate on a cylinder, obtained by threading no or one vison through the hole of the cylinder.

The details of the chargon motion are determined by the effective Hamiltonians that obtain in the charged sectors. Straightforward calculation shows that the presence of the chargon induces a weakening of the background on the bonds and plaquettes that are connected to 


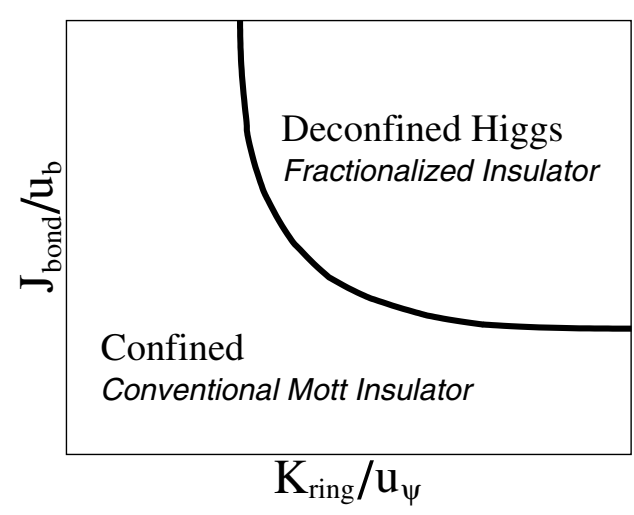

FIG. 3. Diagram of the large $U$ insulating phases of the boson model Eq. (1) in two dimensions. The effective Hamiltonian Eq. (3) is equivalent to the $(2+1) \mathrm{D}$ compact QED gauge theory coupled to a charge 2 scalar. The Mott insulator is conventional or fractionalized depending on whether the effective gauge theory is confined or deconfined.

it: $J_{\text {bond }}^{\prime}=\frac{1}{4} J_{\text {bond }}, K_{\text {ring }}^{\prime}=\frac{5}{8} K_{\text {ring. }}$. Of course, chargon confinement/deconfinement is controlled entirely by the bulk $J_{\text {bond }}, K_{\text {ring }}$ vs $u_{b}, u_{\psi}$ terms that obtain far away from the chargon location and is as expected by looking at $H_{\mathrm{eff}}^{(0)}$ only.

Ground state wave functions and topological order-A good caricature for the ground state wave function of $H_{\text {eff }}^{(0)}$, Eq. (3), is obtained by "Gutzwiller" projecting a superfluid state into the sector $N_{r}=0$,

$$
|\Phi\rangle=P_{0}\left|\theta_{r}=\phi_{r r^{\prime}}=0\right\rangle=\sum_{\left\{n_{r}^{b}, n_{r r^{\prime}}^{\psi}\right\}}^{\prime}\left|\left\{n_{r}^{b}, n_{r r^{\prime}}^{\psi}\right\}\right\rangle,
$$

where the last form is written in the boson number basis and the primed sum is over all configurations such that $N_{r}=0$ at every site $r$. This is the exact ground state wave function when $u_{b}=u_{\psi}=0$ but is not normalizable. A normalizable wave function is obtained by introducing a cutoff for large occupation numbers at each site as is appropriate for nonzero $u_{b}, u_{\psi}$. Below, we leave any such cutoff procedure implicit.

A topologically distinct ground state on a cylinder is obtained by Gutzwiller projecting a superfluid state with one vortex threading the cylinder [14]:

$$
\left|\Phi_{v}\right\rangle=\sum_{\left\{n_{r}^{b}, n_{r r^{\prime}}\right\}}^{\prime}(-1)^{N_{\mathrm{col}}^{\psi}}\left|\left\{n_{r}^{b}, n_{r r^{\prime}}^{\psi}\right\}\right\rangle,
$$

where $N_{\text {col }}^{\psi}$ is a sum of $n_{r r^{\prime}}^{\psi}$ in a given columnar "cut" of $\hat{\mathbf{x}}$-directed links (assuming the cylinder is defined by periodic boundary conditions along $\hat{\mathbf{x}}$ ). Because of the constraints, the parity of $N_{\mathrm{col}}^{\psi}$ is the same for all columns so that the location of the cut is arbitrary. The projected vortex state describes one vison threading the hole of the cylinder.

The presence of topological order is established by noticing that the normalized overlap $\left\langle\Phi \mid \Phi_{v}\right\rangle /\langle\Phi \mid \Phi\rangle$ goes to zero as $O\left(e^{-c L_{y}}\right)$ with the system size [15], and that all local physical operators are the same in the two states since the column defining $N_{\text {col }}^{\psi}$ can be deformed away from any such operator. Thus, the states with no or one vison are indeed orthogonal to each other and degenerate in the thermodynamic limit.

The boson Hamiltonian Eq. (1) is unfrustrated, in the sense that the hopping amplitudes are all positive. It is well known then that the ground state wave function is unique and has positive amplitudes in the boson number basis. This does not contradict the topological order in the fractionalized state. The column parity $(-1)^{N_{\text {col }}^{\psi}}$ is conserved by the Hamiltonian $H_{\text {eff }}^{(0)}$, and the theorem applies to $H_{\text {eff }}^{(0)}$ only separately in the even and odd sectors on the cylinder. By taking the combinations $|\Phi\rangle \pm\left|\Phi_{v}\right\rangle$, we indeed obtain positive wave functions that reside completely in the even or odd sectors. As far as the bare boson Hamiltonian Eq. (1) is concerned, it is more appropriate to speak of the states with no or one vison.

Variations in 2D.-A simple variation of the model considerably enhances the region of stability of the fractionalized phase. Consider "half filling" for the site bosons described by the modified Hubbard repulsion terms,

$$
H_{u_{b}, U}=u_{b} \sum_{r}\left(n_{r}^{b}-\frac{1}{2}\right)^{2}+U \sum_{r}\left(N_{r}-1\right)^{2}
$$

All other terms are unchanged. In the large $U$ limit, the corresponding compact QED theory now has static charges \pm 1 placed on the $A$ and $B$ sublattices, respectively [4], and is at half filling for the gauge charge 2 matter field. The fractionalized insulator now occupies a larger area and extends all the way to infinitesimally small $J_{\text {bond }} / u_{b}$ for infinitely large $K_{\text {ring }} / u_{\psi}$ as shown in Fig. 4. It is also more stable for large $J_{\text {bond }} / u_{b}$ due to frustration coming from the Berry phase terms in the corresponding Ising gauge theory [16].

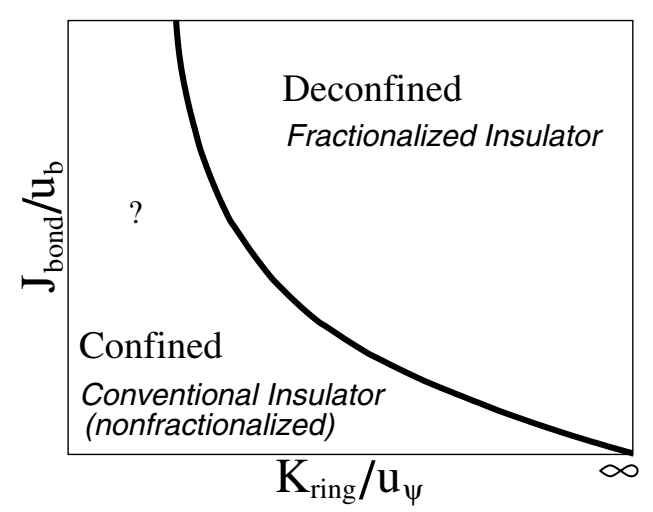

FIG. 4. Large $U$ insulating phases of the $2 \mathrm{D}$ boson model at half filling, Eq. (6). The region of stability of the fractionalized phase is enhanced compared with Fig. 3 at integer filling. There may be several (?) nonfractionalized insulating states with broken translational invariance; here, we focus on the fractionalized state only. 


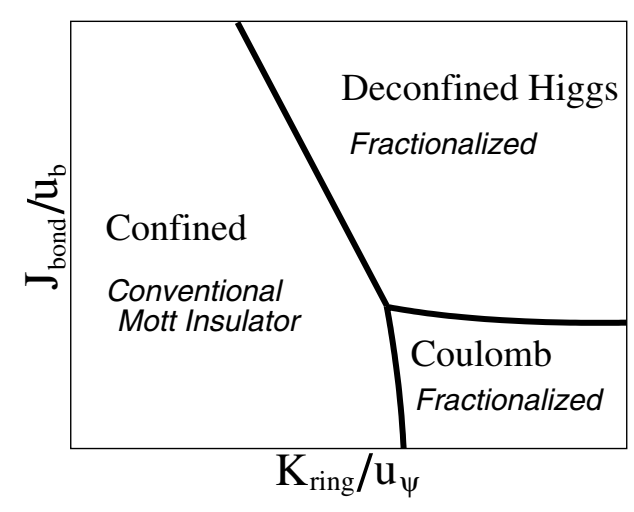

FIG. 5. Same as in Fig. 3 but in three dimensions. There is an additional fractionalized phase, the "Coulomb" phase, with the distinct excitations being a gapped charge $q_{b} / 2$ chargon, gapless photon, and a gapped monopole.

In the model Eq. (1), chargons are bosonic excitations. However, as noted above, the background couplings are weakened in the vicinity of a chargon. It is then plausible that the corresponding $K_{\text {ring }}^{\prime}<0$ in some model, in which case it is energetically favorable for a vison to bind to the chargon thus forming a fermionic excitation. This possibility is indeed realized when we modify our model slightly by allowing some frustration in hopping [15]. Thus, we obtain an explicit boson model that has an insulating phase with fermionic excitations carrying fractional charge. If this unusual insulator is doped, we would obtain a Fermi liquid, i.e., a metallic phase in a boson model, a true Bose metal.

Three dimensions. - Consider now the 3D version of our boson model on a bond-centered cubic lattice. Proceeding as before, the large $U$ Mott insulating states are described by the effective Hamiltonian $H_{\text {eff }}^{(0)}$, Eq. (3), which is equivalent to the $(3+1) \mathrm{D}$ version of the compact $U(1)$ gauge theory coupled to a charge 2 scalar. The phase diagram is shown in Fig. 5, and now has three distinct phases. The "confined" phase is the conventional Mott insulator. The deconfined Higgs phase is similar to the fractionalized phase in two dimensions. The distinct excitations here are gapped charge $q_{b} / 2$ chargon and neutral $Z_{2}$ vortex loop (vison). The vison excitation energy is proportional to the loop length, and the loops do not proliferate up to a finite temperature. Thus, in this phase in 3D, the topological order is stable for small finite temperature (unlike 2D where a finite density of thermally excited point visons destroys the topological order at any nonzero temperature).

Finally, the "Coulomb" phase is also fractionalized. In this phase, the low-energy theory in the ground state sector is that of the pure gauge $(3+1) \mathrm{D}$ compact QED in its Coulomb phase, and has a gapless linearly dispersing gauge boson (photon) and a gapped topological point defect (monopole) as its distinct excitations. The charged sector has charge $q_{b} / 2$ excitations above the gap $U$, but these now interact via an emergent long-range Coulomb interaction. It is quite surprising that a simple Hamiltonian such as Eq. (1) can have such an unusual phase.

Discussion.-The most intriguing aspect of this paper is the simplicity of the Hamiltonians that realize a variety of unconventional quantum phases, and the possibility that such systems may actually be made in a laboratory.

In $2 \mathrm{D}$, one likely realization may be a Josephson junction array. The particular Hubbard terms can in principle be achieved by controlling the electrostatics of the islands [7,9]. Possible techniques for detecting fractions of charge are discussed in Ref. [17]. Of direct relevance for the implementation of topologically protected qubits is the vison trapping experiment: A $2 \pi$ vortex remains trapped in a hole in the system even when the system is cycled from the superfluid to the fractionalized insulator and back. For this to work, one needs to go to temperatures well below the vison gap. Such flux trapping corresponds directly to the ability of the qubit to retain its state in the experimental environment. In this context, we want to note again that in $3 \mathrm{D}$, unlike $2 \mathrm{D}$, the topological order is not destroyed by small finite temperature.

We thank P. A. Lee, A. Vishwanath, and X.-G. Wen for useful discussions. This work was supported by the MRSEC program of the National Science Foundation under Grant No. DMR-9808941.

[1] N. Read and S. Sachdev, Phys. Rev. Lett. 66, 1773 (1991); X.-G. Wen, Phys. Rev. B 44, 2664 (1991).

[2] R. Moessner and S. L. Sondhi, Phys. Rev. Lett. 86, 1881 (2001).

[3] L. Balents, M. P. A. Fisher, and S. M. Girvin, cond-mat/ 0110005.

[4] T. Senthil and O. I. Motrunich, cond-mat/0201320.

[5] P.W. Anderson, Science 235, 1196 (1987); S. Kivelson, D. S. Rokhsar, and J. Sethna, Phys. Rev. B 35, 8865 (1987); T. Senthil and M. P. A. Fisher, Phys. Rev. B 62, 7850 (2000).

[6] A. Kitaev, quant-ph/9707021.

[7] L. B. Ioffe et al., Nature (London) 415, 503 (2002).

[8] X.-G. Wen, Phys. Rev. Lett. 88, 011602 (2002).

[9] Electrostatics that favors charge neutrality in each cluster can be realized, e.g., as in the "JJK" model of Ref. [7], with which our 2D model bears close resemblance.

[10] S. L. Sondhi, S. M. Girvin, J. P. Carini, and D. Shahar, Rev. Mod. Phys. 69, 315 (1997).

[11] E. Fradkin and S. H. Shenker, Phys. Rev. D 19, 3682 (1979).

[12] X.-G. Wen, Int. J. Mod. Phys. B 4, 239 (1990).

[13] T. Senthil and M. P. A. Fisher, Phys. Rev. B 63, 134521 (2001).

[14] D. A. Ivanov and T. Senthil, cond-mat/0204043.

[15] O. I. Motrunich and T. Senthil (unpublished).

[16] S. Sachdev and M. Vojta, J. Phys. Soc. Jpn. 69, 1 (2000).

[17] T. Senthil and M. P. A. Fisher, Phys. Rev. Lett. 86, 292 (2001); Phys. Rev. B 64, 214511 (2001). 